\title{
Nonlinear Frequency Response Analysis of Structures Based on Eigenvector Perturbation
}

\author{
Yuan Wei ${ }^{1, \mathrm{a}}$, Dai $\mathrm{Xu}^{2, \mathrm{~b}}$, Longbin Liu ${ }^{1, \mathrm{c}}$, Weihua Zhang ${ }^{1, \mathrm{~d}_{*}}$ \\ ${ }^{1}$ National University of Defense Technology, College of Aerospace and Engineering, Changsha 410073, \\ China \\ ${ }^{2}$ China Aerospace Science and Technology Corporation, Beijing 100048, China \\ aemail: \\ wei-0330@163.com, bemail: tassadar0330@163.com,'email: longbuaa@163.com, \\ _kjs@163.com \\ *corresponding author
}

Keywords: Nonlinear normal modes (NNMs), describing function (DF), eigenvector perturbation.

\begin{abstract}
This paper proposed a solution method for the nonlinear frequency response analysis of structures combining the Nonlinear Single Resonant Mode (NSRM) theory and eigenvector perturbation theory. The NSRM theory assumes that only the mode in the vicinity of a resonant mode has nonlinear behavior, and the non-resonant mode can be expressed by a linear combination of a set of associated linear normal modes. The describing function (DF) method is used to evaluate the generalized quasilinear matrix, which is treated as a perturbation of the underlying linear system. Finally, a numerical example is presented to show the accuracy of the proposed method.
\end{abstract}

\section{Introduction}

The nonlinear modal superposition method, which is considered as one of the most attractive approach for large-scale structures. The concept of nonlinear normal modes (NNMs) was firstly introduced by Rosenberg[1]. And then, the nonlinear single resonant mode (NSRM) method was developed by [2]-[5] to approximate the solution in the vicinity of a resonant mode. This method gives satisfactory results for the case in which t there is no other mode closes to the resonant mode. Recently, the method is used by Ahmadian and Zamani[6] to identify the nonlinear boundary effects of a cantilever. The essence of NSRM is the assumption that the mode of vibration in the resonant condition is close to the nonlinear normal mode and only one mode has nonlinear behavior, and the participation of the non-resonant modes is relatively small and can be expressed by the linear modal parameters. Furthermore, the nonlinear mode can be expressed as a linear combination of a set of associated linear normal modes. The component of each linear mode is called nonlinear mode participation factor[4].

The describing function (DF) method[7] is used to evaluate the generalized quasilinear matrix, which can be treated as a perturbation of the underlying linear system. Therefore, the eigenvalue perturbation theory[8] is adopted to approximate nonlinear mode participation factors.

\section{Theories}

\subsection{Equation of Motion}

The equation of motion for an N-degree-of-freedom nonlinear system subjected to a harmonic excitation can be generally expressed as

$$
\mathbf{M} \ddot{\mathbf{x}}+\mathbf{C} \dot{\mathbf{x}}+\mathbf{K x}+\mathbf{f}_{n l}(\dot{\mathbf{x}}, \mathbf{x})=\mathbf{q}
$$

where $\mathbf{M}, \mathbf{C}, \mathbf{K}$ are the mass, stiffness and damping matrices, respectively; $\ddot{\mathbf{x}}, \dot{\mathbf{x}}$ and $\mathbf{x}$ are the acceleration, velocity and displacement vectors, respectively; $\mathbf{f}_{n l}(\dot{\mathbf{x}}, \mathbf{x})$ is the nonlinear force vector and $\mathbf{q}$ is the harmonic excitation force vector. 


\subsection{Nonlinear Single Resonant Method}

According to the NSRM theory, in the vicinity of rth resonant mode the solution of equation (1) can be expressed as [4][5]

$$
\mathbf{x}=\tilde{\boldsymbol{\varphi}}_{r} u_{r}(t)+\sum_{\substack{k=1 \\ k \neq r}}^{n} \boldsymbol{\varphi}_{k} u_{k}(t)
$$

where $\tilde{\boldsymbol{\varphi}}_{r}$ is the $r$ th nonlinear eigenvector, $\boldsymbol{\varphi}_{k}$ is the $k$ th linear eigenvector and $u_{k}$ is the $k$ th modal amplitude. The above equation can also be rewritten in a matrix form as

$$
\mathbf{x}=\tilde{\mathbf{\Phi}} \mathbf{u}
$$

where $\tilde{\boldsymbol{\Phi}}=\left[\boldsymbol{\varphi}_{1}, \cdots, \tilde{\boldsymbol{\varphi}}_{r}, \cdots, \boldsymbol{\varphi}_{n}\right]$

Without loss of generality, the nonlinear eigenvector $\tilde{\varphi}_{r}$ can be expressed as a linear combination of linear eigenvectors

$$
\tilde{\boldsymbol{\varphi}}_{r}=\sum_{k=1}^{n} \boldsymbol{\varphi}_{k} b_{k}=\mathbf{\Phi} \mathbf{b}
$$

where $b_{k}$ is the nonlinear mode participation factor ${ }^{[4]} \boldsymbol{\Phi}$ is the mode matrix of underlying linear system. Substituting equation(3) into equation(1) and premultiplying with $\tilde{\boldsymbol{\Phi}}^{T}$ yield

$$
\tilde{\mathbf{M}} \ddot{\mathbf{i}}+\tilde{\mathbf{C}} \dot{\mathbf{u}}+\tilde{\mathbf{K}} \mathbf{u}+\tilde{\boldsymbol{\Phi}}^{T} \mathbf{f}_{n l}(\dot{\mathbf{x}}, \mathbf{x})=\tilde{\boldsymbol{\Phi}}^{T} \mathbf{q}
$$

where

$$
\tilde{\mathbf{M}}=\tilde{\boldsymbol{\Phi}}^{T} \mathbf{M} \tilde{\boldsymbol{\Phi}}, \tilde{\mathbf{C}}=\tilde{\boldsymbol{\Phi}}^{T} \mathbf{C} \tilde{\boldsymbol{\Phi}}, \tilde{\mathbf{K}}=\tilde{\boldsymbol{\Phi}}^{T} \mathbf{K} \tilde{\boldsymbol{\Phi}}
$$

Converting equation (5) into frequency domain, one has

$$
\left(-\Omega^{2} \tilde{\mathbf{M}}+j \Omega \tilde{\mathbf{C}}+\tilde{\mathbf{K}}+\tilde{\boldsymbol{\Phi}}^{T} \mathbf{K}_{n l}(\mathbf{X}) \tilde{\boldsymbol{\Phi}}\right) \mathbf{U}=\tilde{\boldsymbol{\Phi}}^{T} \mathbf{Q}
$$

where $\mathbf{K}_{n l}$ is generalized quasilinear matrix which is evaluated by DF method ${ }^{[9]} \mathbf{K}_{n l}$ relates to $\mathbf{U}$, and excitation frequency $\Omega$ if the nonlinear force depends on velocity $\dot{\mathbf{x}}$.

\subsection{Eigenvector Perturbation Theory}

The solution to the generalized eigenvalue problem is given as

$$
\begin{aligned}
& \boldsymbol{K} \boldsymbol{\varphi}=\lambda \boldsymbol{M} \boldsymbol{\varphi} \\
& \boldsymbol{\varphi}^{T} \boldsymbol{M} \boldsymbol{\varphi}=\boldsymbol{I}
\end{aligned}
$$

where $\mathbf{M}, \mathbf{K}$ are the mass, stiffness matrices; $\lambda, \boldsymbol{\varphi}$ are eigenvalues and eigenvectors.

Suppose that $\lambda_{i}$ and $\boldsymbol{\varphi}_{i}$ are the $i$ th eigenvalue and eigenvector, thus we have

$\boldsymbol{K} \boldsymbol{\varphi}_{i}=\lambda_{i} \boldsymbol{M} \boldsymbol{\varphi}_{i}$

Assume that $\Delta \boldsymbol{M}$ and $\Delta \boldsymbol{K}$ are small perturbations of matrices $\boldsymbol{M}$ and $\mathbf{K}$

$$
\begin{aligned}
\overline{\boldsymbol{M}} & =\boldsymbol{M}+\Delta \boldsymbol{M} \\
\overline{\boldsymbol{K}} & =\boldsymbol{K}+\Delta \boldsymbol{K}
\end{aligned}
$$

It is expected that the new eigenvalues and eigenvectors to be similar to the original, plus small perturbations

$$
\begin{aligned}
& \bar{\lambda}_{i}=\lambda_{i}+\Delta \lambda_{i} \\
& \overline{\boldsymbol{\varphi}}_{i}=\boldsymbol{\varphi}_{i}+\Delta \boldsymbol{\varphi}_{i}
\end{aligned}
$$

According to the first order eigenvalue perturbation theory ${ }^{[8]}$

$$
\begin{aligned}
& \Delta \lambda_{i}^{(1)}=\boldsymbol{\varphi}_{i}^{T}\left(\Delta \boldsymbol{K}-\lambda_{i} \Delta \boldsymbol{M}\right) \boldsymbol{\varphi}_{i} \\
& \Delta \boldsymbol{\varphi}_{i}^{(1)}=\sum_{j=1}^{n} \beta_{i j}^{(1)} \boldsymbol{\varphi}_{j}
\end{aligned}
$$

where, the parameter $\beta_{i j}^{(1)}$ can be calculated by 


$$
\beta_{i j}^{(1)}=\left\{\begin{array}{c}
\frac{1}{\lambda_{i}-\lambda_{j}} \boldsymbol{\varphi}_{i}^{T}\left(\Delta \boldsymbol{K}-\lambda_{i} \Delta \boldsymbol{M}\right) \boldsymbol{\varphi}_{j}, j \neq i, j=1,2, \cdots, n \\
-\frac{1}{2} \boldsymbol{\varphi}_{i}^{T} \Delta M \boldsymbol{\varphi}_{j}, j=i
\end{array}\right.
$$

\subsection{Combination of Eigenvector Perturbation and NSRM}

By neglecting the off-diagonal items in equation(6) and rearranging in terms of reacceptance, the following equation is obtained

$$
\mathbf{P}=\left[\begin{array}{ccccc}
\frac{1}{\Lambda_{11}} & & & & \\
& \ddots & & & \\
& & \frac{1}{\tilde{\Lambda}_{r r}} & & \\
& & & \ddots & \\
& & & & \frac{1}{\Lambda_{n n}}
\end{array}\right] \tilde{\boldsymbol{\Phi}}^{T} \mathbf{F}
$$

Where $\Lambda_{s s}=-\Omega^{2}+j \Omega C_{s}+\omega_{s}^{2}+\boldsymbol{\varphi}_{s}^{T} \mathbf{K}_{n l} \boldsymbol{\varphi}_{s}, s=1,2 \cdots n ; s \neq r$

$$
\tilde{\Lambda}_{r r}=\left(-\Omega^{2} \sum_{k=1}^{n} b_{k}^{2}+j \Omega \sum_{k=1}^{n} b_{k}^{2} c_{k}+\sum_{k=1}^{n} b_{k}^{2} \omega_{k}^{2}\right)+\mathbf{b}^{T} \mathbf{\Phi}^{T} \mathbf{K}_{n l} \mathbf{\Phi} \mathbf{b}
$$

Because it is assumed that the contribution of non-resonant mode is relative small, the term $\boldsymbol{\varphi}_{s}^{T} \mathbf{K}_{n l} \boldsymbol{\varphi}_{s}$ can also be neglected as suggested in [4]. This simplification is followed in this paper in the subsequent discussion. Pre-multiplying both sides of equation(16) by $\tilde{\boldsymbol{\Phi}}$ and combining equation(3), one obtains

$$
\mathbf{X}=\tilde{\mathbf{\Phi}}\left[\begin{array}{ccccc}
\frac{1}{\Lambda_{11}} & & & & \\
& \ddots & & & \\
& & \frac{1}{\tilde{\Lambda}_{r r}} & & \\
& & & \ddots & \\
& & & & \frac{1}{\Lambda_{n n}}
\end{array}\right] \tilde{\mathbf{\Phi}}^{T} \mathbf{F}
$$

From another point of view, the generalized quasilinear matrix $\mathbf{K}_{n l}$ can be treated as a perturbation of the underlying linear system. Applying equation(15), the nonlinear mode participation factors can be approximated by

$$
\begin{gathered}
b_{k} \approx \frac{1}{\omega_{r}^{2}-\omega_{k}^{2}} \boldsymbol{\varphi}_{r}^{T} \mathbf{K}_{n l} \boldsymbol{\varphi}_{k}, k=1 \cdots n, k \neq r \\
b_{r} \approx 1
\end{gathered}
$$

Substituting equation(18) into equation(17) yields a set of complex algebraic equations . It can be solved using the Newton-Raphon method.

\section{Numerical Example}

Consider a mass-spring system with 5 DOFs as illustrated in figure 1. The mass, damping, and stiffness are $m_{1} \sim m_{5}=1 \mathrm{~kg}, c_{1} \sim c_{5}=0.03 \mathrm{~N} \mathrm{sec} / \mathrm{m}, k_{1} \sim k_{5}=100 \mathrm{~N} / \mathrm{m}$, respectively. The third spring is assumed to be nonlinear, whose characteristic is given by 
$f_{n l}(y)=\alpha y^{3}$

Where the nonlinear coefficient $\alpha=2.4 \mathrm{~N} / \mathrm{m}^{3}$.

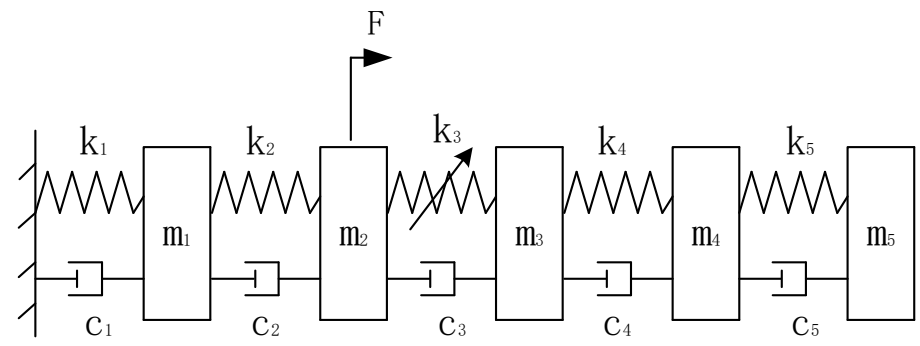

Fig.1. Model of the 5-DOF system

The harmonic excitation force $F=2 \cos (\Omega t)$ is applied on $m_{2}$. Figure 2 shows the frequency response of $m_{1}$; figure 3 and 4 give the frequency response in detail in the vicinity of the first and second modes respectively. The results suggest that the proposed method has a good agreement with the harmonic balance method.

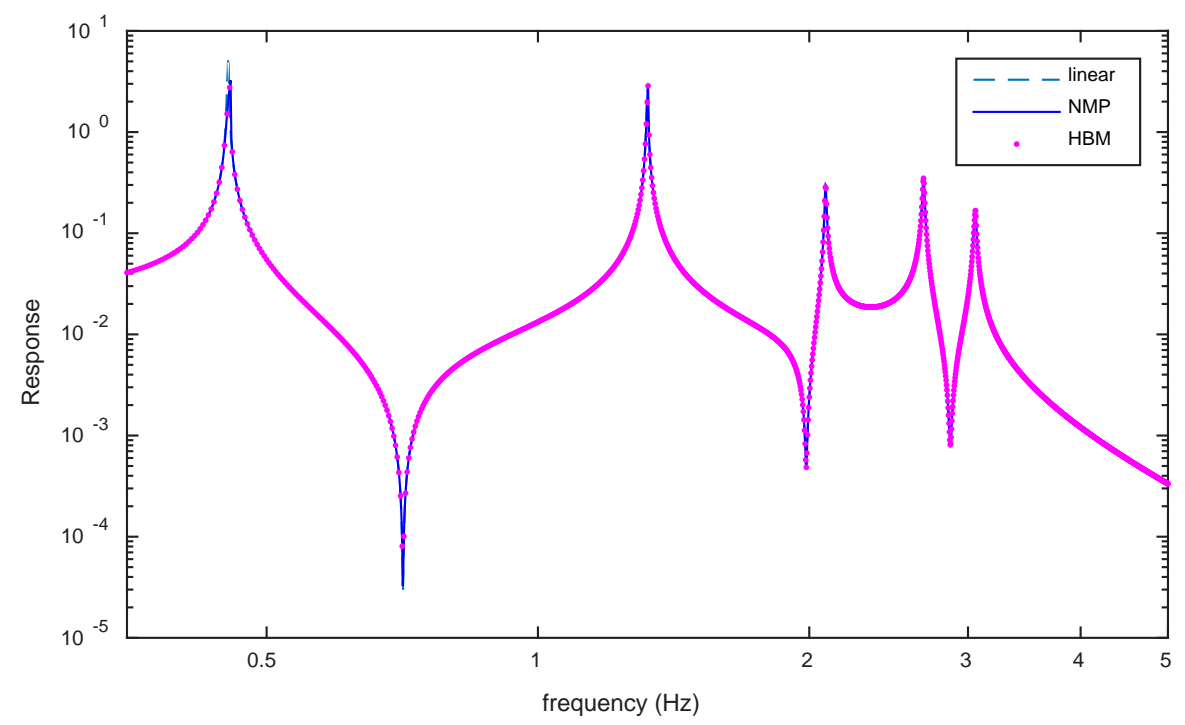

Fig. 2. Frequency response of $m_{1}$

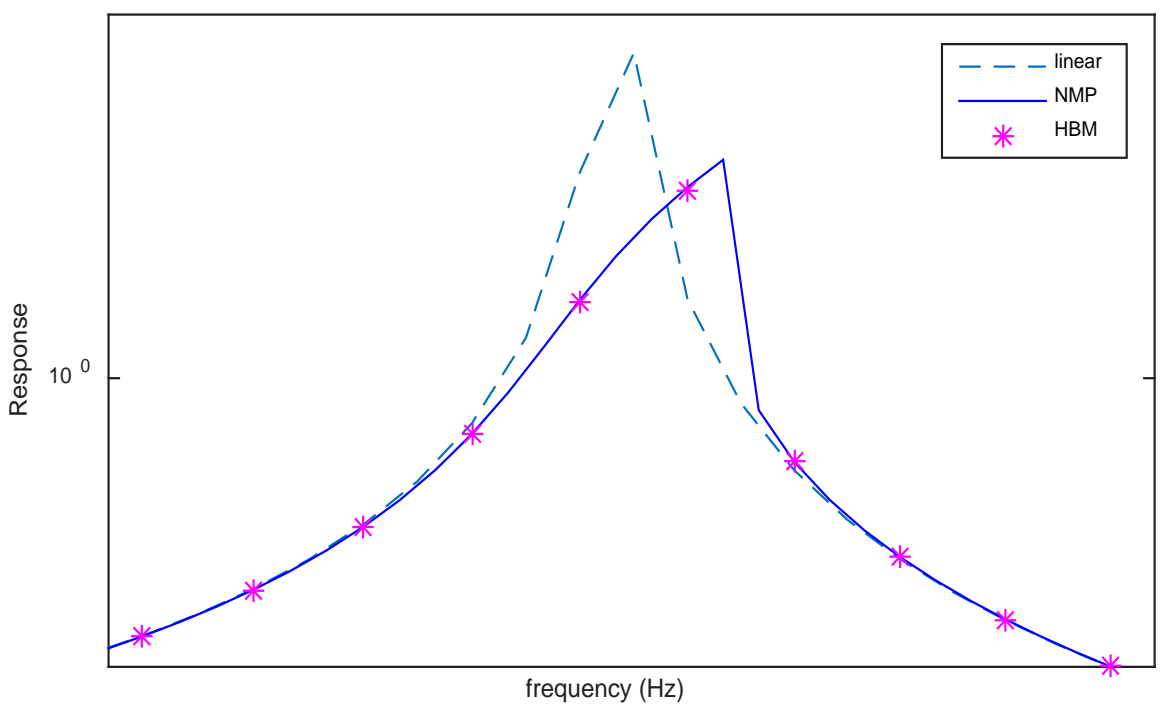

Fig. 3. Frequency response of $\mathrm{m}_{1}$ (the first mode in detail) 


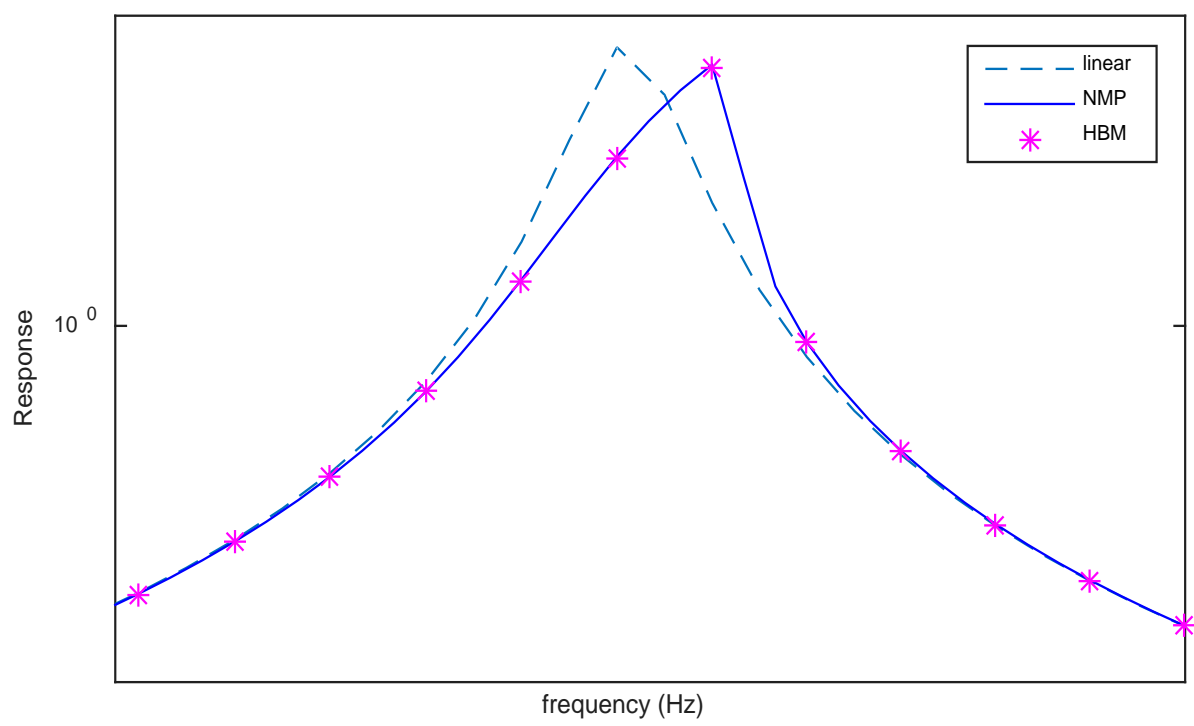

Fig. 4. Frequency response of $\mathrm{m}_{1}$ (the second mode in detail)

\section{Conclusion}

An efficient solution method for the nonlinear frequency response analysis of structures is proposed, which combined the describing function method, nonlinear single resonant mode and eigenvector perturbation. The nonlinear stiffness matrix, which is evaluated by describing function method, is treated as a perturbation of the underlying linear system. As a simple numerical example, a 5-DOF mass and spring system is analyzed and the results are compared with harmonic balance method. The results show that the proposed method has a good agreement with the harmonic balance method.

\section{Acknowledgement}

In this paper, the research was sponsored by the Research project of National University of Defense Technology (Project No. ZK16-03-33).

\section{References}

[1] R. M. Rosenberg, (1962) The normal modes of non-linear n-degree-of-freedom systems[J], ASME, Journal of Applied Mechanics, 29, 7-14.

[2] W. Szemplińska-Stupnicka, (1979) The modified single mode method in the investigations of the resonant vibration of nonlinear systems[J], Journalof Sound and Vibration, 63, 475-489.

[3] W. Szemplińska-Stupnicka, (1983) Nonlinear normal modes and the generalized Ritz method in the problems of vibrations of nonlinear elastic continuous systems[J], International Journal of Non-linear Mechanics, 18, 149-165.

[4] S. Setio, H. D. Setio, and L. Jezequel, (1992) A method of nonlinear modal identification from frequency response tests[J], Journal of Sound and Vibration, 158, 497-515.

[5] A. S. Nobari, and M. Shahramyar, (2003) Improvement of nonlinear single resonant mode method, ASME[J], Journal of vibration and Acoustics, 125, 59-63.

[6] H. Ahmadian, A. Zamani, (2009) Identification of nonlinear boundary effects using nonlinear normal modes[J], Mechanical System and signal processing, 23, 2008-2018. 
[7] O. Tanrikulu, B. Kuran, N. Ozguven, M. Imregun. (1993) Forced Harmonic Response Analysis of Nonlinear Structures Using Describing Functions[J], AIAA Journal. 31(7), 1313-1320

[8] B. B. William, (1987) An improved computational technique for perturbations of the generalized symmetric linear algebraic eigenvale problem[J], Int. J. Numer. Method in Eng., 24, 529-541.

[9] Stewart, Columba; Stewart, G. W. Ji-Guang Sun, (1990) Matrix Perturbation Theory[M], Academic Press INC. 